\title{
Establishment of a Methodology for the Determination of Mercury in Environmental Samples by Potentiometric Stripping Analysis (PSA)
}

\author{
Mario L. Miranda ${ }^{a}$, Carlos L. Rojas ${ }^{b, *}$ \\ ${ }^{a}$ Laboratorio de Análisis y Servicios Fisicoquímicos, Facultad de Ciencias Naturales y \\ Exactas, Universidad Autónoma de Chiriquí, Estafeta Universitaria, David, Chiriquí, Republica \\ de Panamá \\ ${ }^{\mathrm{b}}$ Escuela de Química, Centro de Electroquímica y Energía Química, Universidad de Costa \\ Rica, 2060 San Pedro Montes de Oca, Costa Rica
}

Received 28 May 2004; accepted in revised form 22 September 2004

\begin{abstract}
The objective of this paper is the establishment of the better instrumental conditions of analysis for the determination and quantification of mercury in environmental samples by potentiometric stripping analysis. This determination is based in the deposition of mercury into a thin gold film, previously deposited onto a glassy carbon electrode, and then stripped back to the work solution by means of an added oxidant agent. We carried out tests to determine the oxidant concentration, electrolysis potential, support electrolyte and its concentration, potassium chloride concentration and the detection / quantification limits, having in mind to determine in a precise, reliable and reproducible form the presence of mercury, its further quantification, previous mineralization by microwave digestion and its preconcentration by cation exchange column chromatography, to eliminate interferences from metallic ions. The quantification was carried out by the standard additions method. This work shows a detection limit of 5 $\mu \mathrm{g} / \mathrm{L}$ and quantification limit of $17 \mu \mathrm{g} / \mathrm{L}$.
\end{abstract}

Keywords: mercury, stripping, PSA, environment, optimization, gold film, detection limits.

\section{Introducción}

La contaminación ambiental por mercurio es una preocupación mundial desde hace décadas, toda vez que este elemento es sumamente toxico, ocasiona daños al hígado, al sistema nervioso central y severas deficiencias intelectuales.

\footnotetext{
Corresponding author. E-mail address: cleon@equi.ucr.ac.cr
} 
Mas peligroso aún resulta el hecho de que bajo condiciones anóxicas puede ser metabolizado por bacterias en metil o dimetil mercurio, las cuales son especies volátiles y lipofílicas que fueron asociadas a la "enfermedad de Minamata" en la decada de los $50^{\prime}[1]$.

El mercurio metálico posee una amplia gama de usos en el ámbito industrial, desde procesos electrolíticos, industria eléctrica, fabricación de instrumentos, y en catálisis química. Este se encuentra como metal traza en muchos minerales en concentraciones de aproximadamente de $80 \mu \mathrm{g} / \mathrm{kg}$ (ppb) [2].

En 1976, Jagner y colaboradores reportaron el desarrollo de una técnica analítica para la determinación de trazas de metales a la cual llamaron Análisis de Redisolución Potenciométrica [3]. Este método consiste de dos pasos fundamentales. En el primer paso ocurre la preconcentración de los metales en la superficie del electrodo de trabajo en cantidades apreciables; este paso es común a todos los métodos de redisolución electroquímica. En el caso del análisis por redisolución potenciométrica el proceso se lleva a cabo bajo agitación constante y con un potencial lo suficientemente negativo como para reducir los cationes metálicos en solución.

$$
\mathrm{Hg}^{2+}+2 e^{-} \rightarrow \mathrm{Au}(\mathrm{Hg})
$$

En el segundo paso, llamado redisolución, los metales previamente depositados vuelven al seno de la disolución; la utilización de un agente oxidante adicionado a la disolución permite la re-oxidación de los cationes metálicos [4,5]. Según la siguiente reacción:

$$
A u(H g)+A j(o x) \rightarrow H g^{2+}+A j(\text { red })
$$

donde Aj representa la especie oxidante.

En la segunda etapa se lleva a cabo la medición del potencial obtenido con respecto al tiempo, de aquí que se conozca esta técnica más apropiadamente como cronopotenciometría [6]. El análisis de redisolución potenciométrica a diferencia de otras técnicas tiene la ventaja de que no presenta interferencias por materia orgánica en la muestra y su mayor desventaja es la elección del 
electrolito soporte adecuado para la obtención de elementos analizados en forma libre [7]. A pesar de ser una técnica de relativamente fácil aplicación es importante observar que existen algunas interferencias reportadas debido a la presencia de rodio(III) y paladio(II), los cuales en concentraciones mayores a los $100 \mu \mathrm{g} / \mathrm{L}$ producen perdida en la sensibilidad del método. De la misma forma la presencia de cobre(II) y selenio(IV) producen desplazamientos de la señal del mercurio, aunque no se reporta perdida de sensibilidad en este caso [8].

Resulta importante señalar que antes de implementar cualquier metodología de análisis, es necesaria la optimización de las condiciones experimentales que determinan la eficiencia del ensayo, debido a que estas presentan variaciones en función de las características propias de cada equipo y de los requerimientos operacionales del laboratorio donde se llevará a cabo la medición [9]. Por esta razón, el objetivo principal de esta investigación es establecer estas condiciones, basándonos en la obtención de las mejores relaciones de sensibilidad y linealidad para cada parámetro analizado, con el fin de reportar datos precisos, reproducibles y confiables. En una etapa posterior, se aplicará la metodología desarrollada a la determinación de mercurio en muestras medioambientales.

\section{Parte experimental}

\section{Instrumental}

Para la obtención de todos los cronopotenciógramas se utilizó una unidad de redisolución potenciométrica Tracelab PSU20 (Radiometer Copenhagen) con una estación de trabajo SAM20 unida a un computador personal por medio de la interfase TAP2 trace talk de la misma marca. Las condiciones de trabajo (concentración de oxidante, potencial de electrólisis, electrolito soporte y su concentración, tiempo de deposito de la película de oro, concentración de $\mathrm{KCl}$ así como los limites de detección y cuantificación) se seleccionaron bajo el criterio de la obtención de las mejores relaciones de sensibilidades y linealidad. El sistema usa tres electrodos: un electrodo de trabajo de carbón vitrificado sobre el cual se deposita una película de oro, un electrodo auxiliar de platino y un electrodo de referencia de calomel. 


\section{Reactivos}

Las disoluciones de trabajo de mercurio de $10 \mathrm{mg} / \mathrm{L}$, fueron preparadas mensualmente a partir de una disolución madre de $1000 \mathrm{mg} / \mathrm{L}$, por dilución con agua desionizada obtenida de un sistema Milli-Q, con una resistividad mayor que 18.2 $\mathrm{M} \Omega$. La disolución de cloruro de oro de $1000 \mathrm{mg} / \mathrm{L}$ fue preparada a partir de un titrisol de la casa Merck. La disolución madre de cloruro de potasio $1 \mathrm{~mol} / \mathrm{L}$ fue preparada a partir del reactivo calidad para análisis. Todos los ácidos minerales utilizados en esta investigación son de calidad ultrapura.

\section{Pre-tratamiento del electrodo de trabajo}

Debido a las características de la técnica, la superficie del electrodo debió ser tratada previamente para garantizar la transferencia apropiada de electrones, la estabilidad mecánica de la película que se depositará sobre éste y la reproducibilidad de las señales obtenidas. Antes de iniciar las determinaciones el electrodo fue pulido utilizando alumina de 0.1 micras. Seguidamente se depositó durante un minuto la película de oro y se sometió el sistema a 10 ciclos de electrólisis/redisolución sobre $20.0 \mathrm{~mL}$ de una disolución que contenía $62.5 \mu \mathrm{g} / \mathrm{L}$ de $\mathrm{Hg}^{2+}$, hasta obtener señales reproducibles a un potencial de $600 \mathrm{mV}$ contra un electrodo de calomel saturado [10].

\section{Preparación de la película de oro}

La película de oro se obtuvo por medio de la inmersión del electrodo de trabajo en una celda conteniendo $20.0 \mathrm{~mL}$ de una disolución de $\mathrm{Au}(\mathrm{III}) 1000 \mathrm{mg} / \mathrm{L}$ y $\mathrm{HCl} 0.04 \mathrm{M}$ con la posterior aplicación de un potencial de $-100 \mathrm{mV}$ [11]. El tiempo requerido para la obtención de una película apropiada es evaluado en esta investigación por lo que en la siguiente sección se presentan los resultados obtenidos. 


\section{Resultados y discusión}

\section{Selección del electrolito soporte y su concentración}

Para seleccionar el electrolito soporte se consideró inicialmente la utilización de $\mathrm{HCl}$, pero este fue descartado debido a la baja estabilidad mecánica y química que presentaba la película de oro al usar este ácido y a que al aumentar la concentración de éste, la sensibilidad de la determinación disminuía drásticamente. Se ensayó con ácido sulfúrico en presencia de concentraciones variables de $\mathrm{KCl}$ como fuente de iones $\mathrm{Cl}^{-}$. Se observó un aumento considerable en la estabilidad mecánica de la película y a pesar del aumento de la concentración del ácido, la perdida de sensibilidad no fue tan pronunciada para este sistema. El mejor electrolito soporte está formado por una disolución de ácido sulfúrico con una concentración de $0.210 \mathrm{~mol} / \mathrm{L}$, debido a que proporcionó la mejor sensibilidad (Fig. 1) y linealidad para la determinación.

Para la optimización de la concentración de $\mathrm{KCl}$ en el electrolito soporte, se varió sistemáticamente la concentración del mismo en la celda y como se observa en la Fig. 2, se obtienen las mejores relaciones de sensibilidad/linealidad cuando la concentración de $\mathrm{KCl}$ es $0.0015 \mathrm{~mol} / \mathrm{L}$, por lo que se seleccionó ésta como la óptima para el resto de las determinaciones.

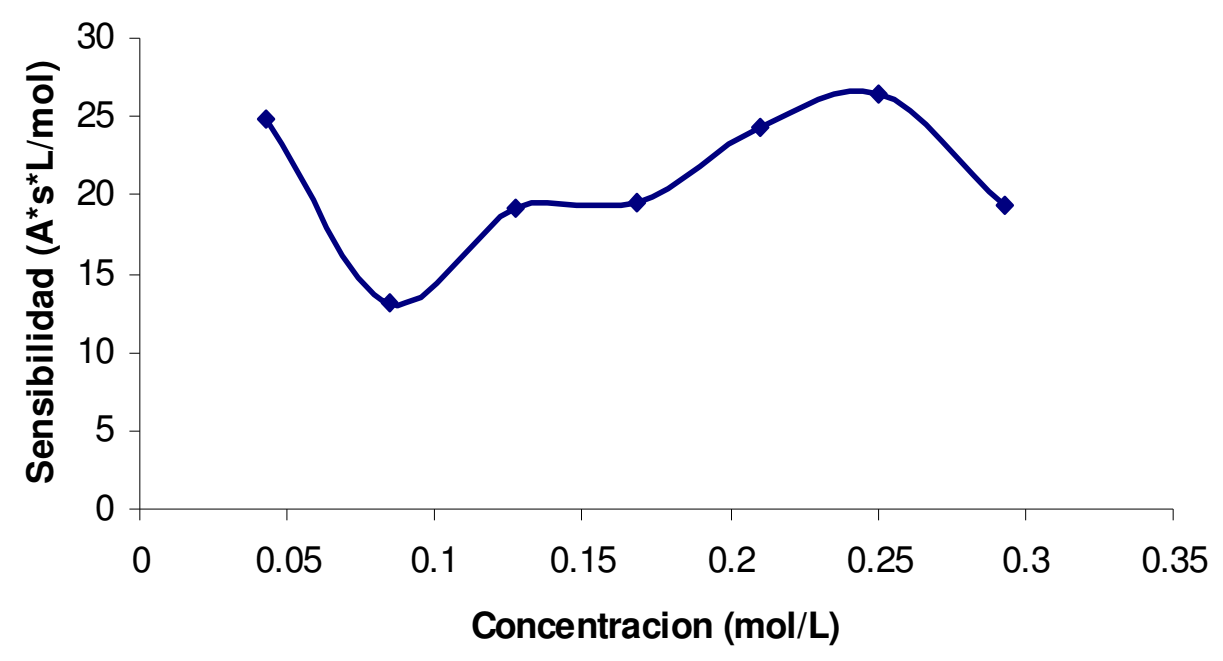

Figura 1. Variación de la sensibilidad del método como función de la concentración de electrolito soporte. 


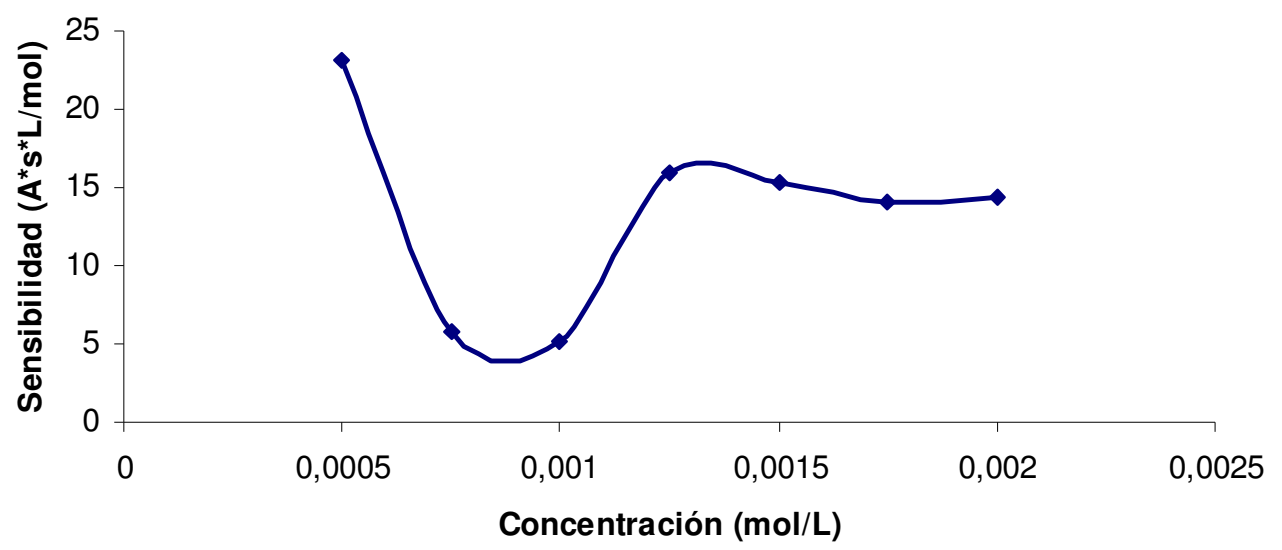

Figura 2. Variación de la sensibilidad del método como función de al concentración de $\mathrm{KCl}$ en celda.

\section{Selección de la concentración de oxidante}

La selección de la concentración de oxidante apropiada para la determinación es de suma importancia, toda vez que este parámetro influye directamente en el proceso de redisolución del mercurio depositado en la película de oro. Para realizar la optimización, sobre $20.0 \mathrm{~mL}$ de una disolución que contiene $62.5 \mu \mathrm{g} / \mathrm{L}$ de $\mathrm{Hg}^{2+}$, se agregaron cantidades variables de la disolución oxidante de $\mathrm{Au}(\mathrm{III})$ de $1000 \mathrm{mg} / \mathrm{L}$. Como se observa en la Fig. 3, el incremento de la concentración de oxidante, incrementa la reproducibilidad de las señales obtenidas, si bien simultáneamente se observa una perdida en la sensibilidad de hasta un 50\% de la señal obtenida con $5.0 \mathrm{mg} / \mathrm{L}$ de oro. Finalmente se seleccionó $25.0 \mathrm{mg} / \mathrm{L}$ de $\mathrm{Au}(\mathrm{III})$ como la concentración óptima de oxidante, pues proporciona la mejor relación de reproducibilidad y sensibilidad.

\section{Selección del potencial de electrólisis}

En la optimización de este parámetro es importante señalar que el ámbito de trabajo para este electrodo esta limitado por la electrólisis del agua, por lo que a potenciales menores a $-300 \mathrm{mV}$, la evolución de hidrógeno compromete la reproducibilidad y la sensibilidad de la determinación. Se realizó un estudio de este parámetro, modificando el potencial de electrólisis desde $-1000 \mathrm{mV}$ hasta 50 $\mathrm{mV}$ a intervalos de $50 \mathrm{mV}$. Se determinó que el potencial que aportaba la mejor 
señal y reproducibilidad se ubicaba a $-950 \mathrm{mV}$. Posteriormente se verificó la existencia de interferencias por la generación visible de hidrógeno sobre el electrodo de trabajo, por lo que se procedió a estudiar los potenciales comprendidos entre $-300 \mathrm{mV}$ y $-150 \mathrm{mV}$. Se seleccionó $-250 \mathrm{mV}$ como el potencial óptimo para realizar las mediciones debido a que proporcionó la mejor sensibilidad en este ámbito, como se observa en la Fig. 4.

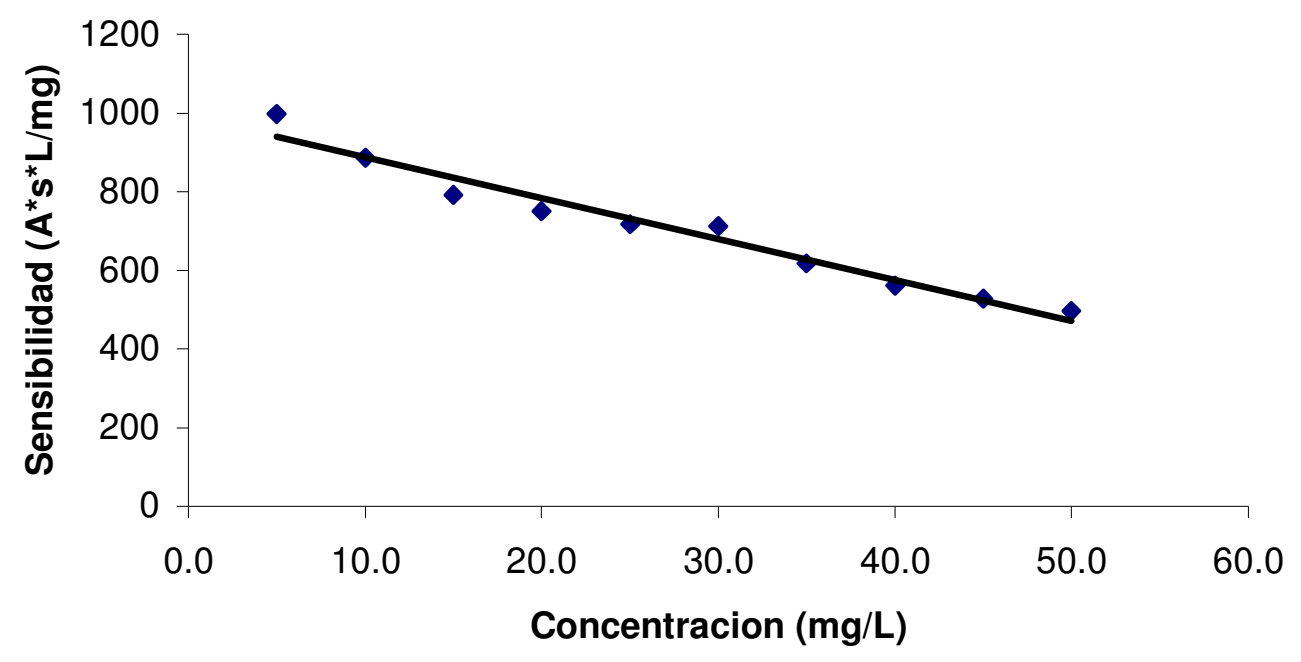

Figura 3. Determinación de la concentración óptima de oxidante en la celda.

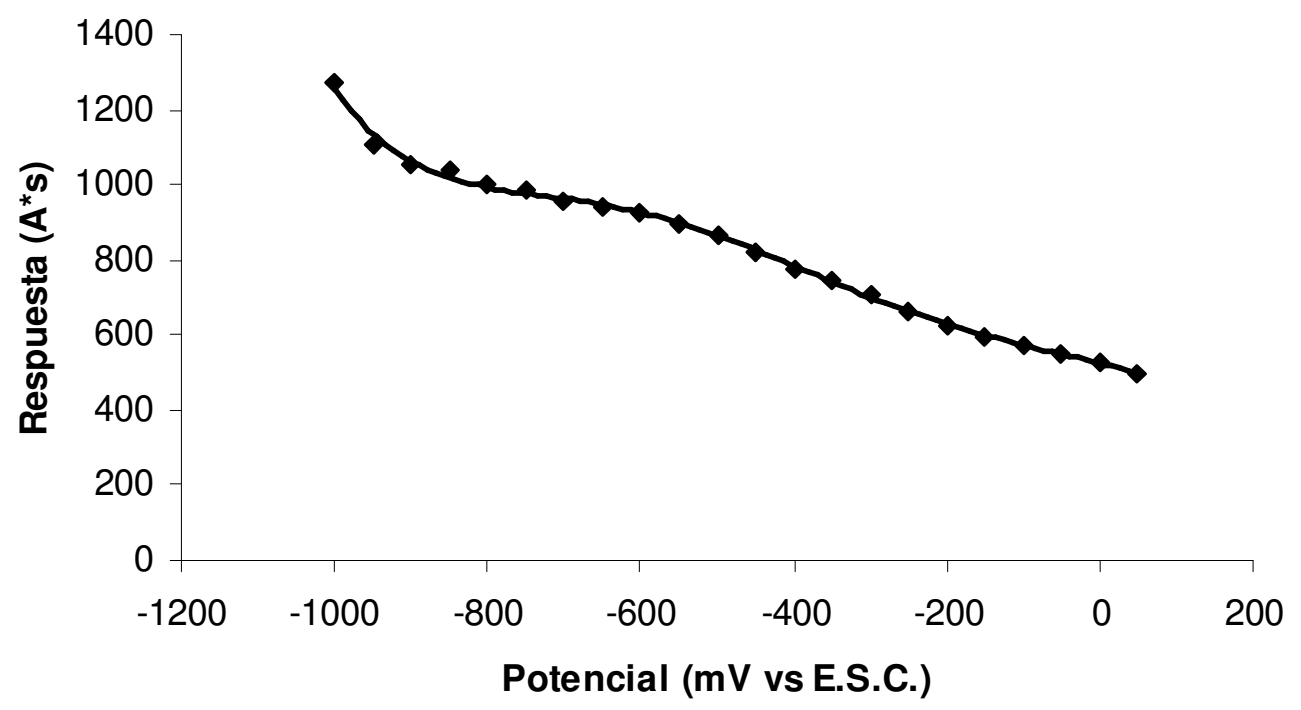

Figura 4. Establecimiento del potencial de electrólisis óptimo para la determinación de mercurio. 


\section{Selección del tiempo de electroplateado para la película de oro}

Para llevar a cabo la optimización de este parámetro se procedió a modificar el tiempo de electroplateado del electrodo de carbón vitrificado, con una disolución de $\mathrm{Au}^{3+}$ de $1000 \mathrm{mg} / \mathrm{L}$. Los tiempos fueron variados entre 60 y 480 segundos. Como se observa en la Fig. 1, a medida que se aumenta el tiempo de electroplateado, la sensibilidad de la determinación aumenta hasta obtenerse un máximo en 240 segundos. Sin embargo la linealidad obtenida en este punto no fue la mejor, por lo que se seleccionó como óptima, la película producida por un tiempo de deposición de $420 \mathrm{~s}$, pues la misma proporcionó la mejor relación entre linealidad y sensibilidad.

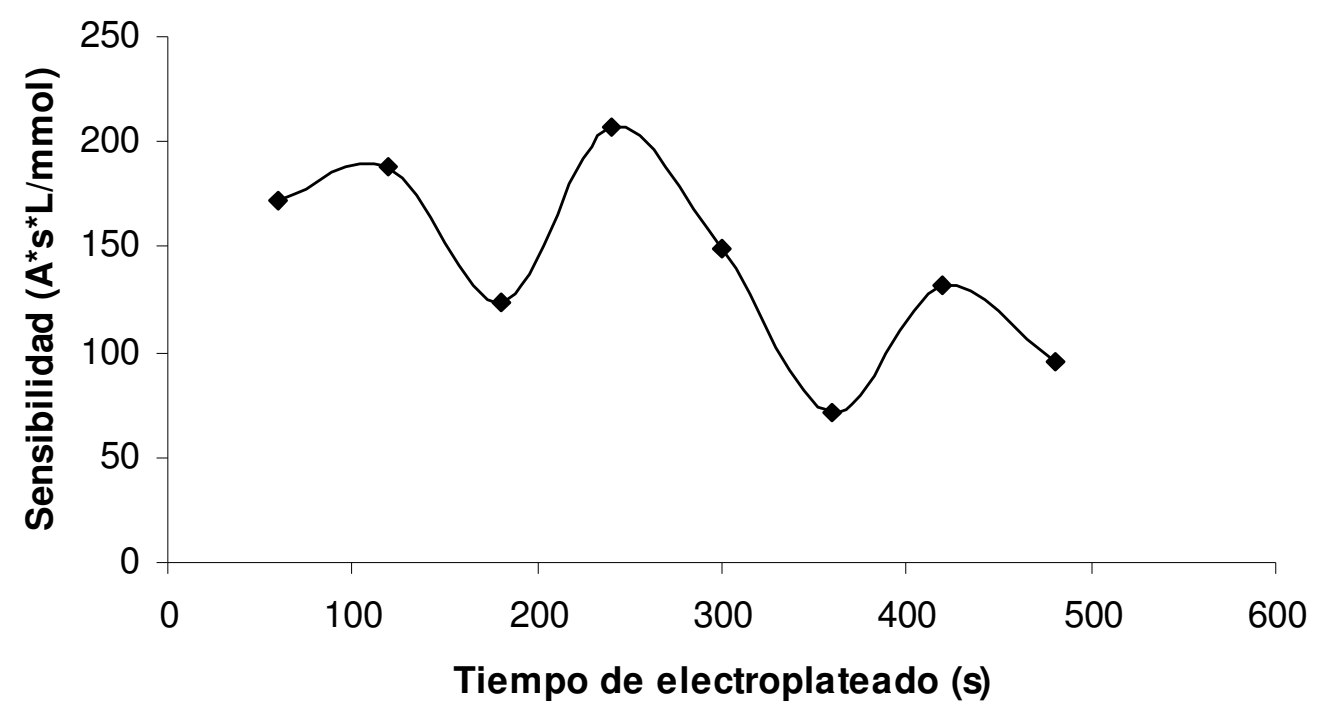

Figura 5. Variación de la sensibilidad del método como función del tiempo de deposición de la película de oro.

\section{Determinación del ámbito de linealidad}

Para la determinación del ámbito de linealidad de este método se utilizó el método de las adiciones estándares. Se hicieron incrementos sucesivos de la concentración de mercurio, en intervalos de $62.5 \mu \mathrm{g} / \mathrm{L}$. Como se observa en la Fig. 6, se encontró un ámbito lineal desde 0 hasta $250 \mu \mathrm{g} / \mathrm{L}$. 


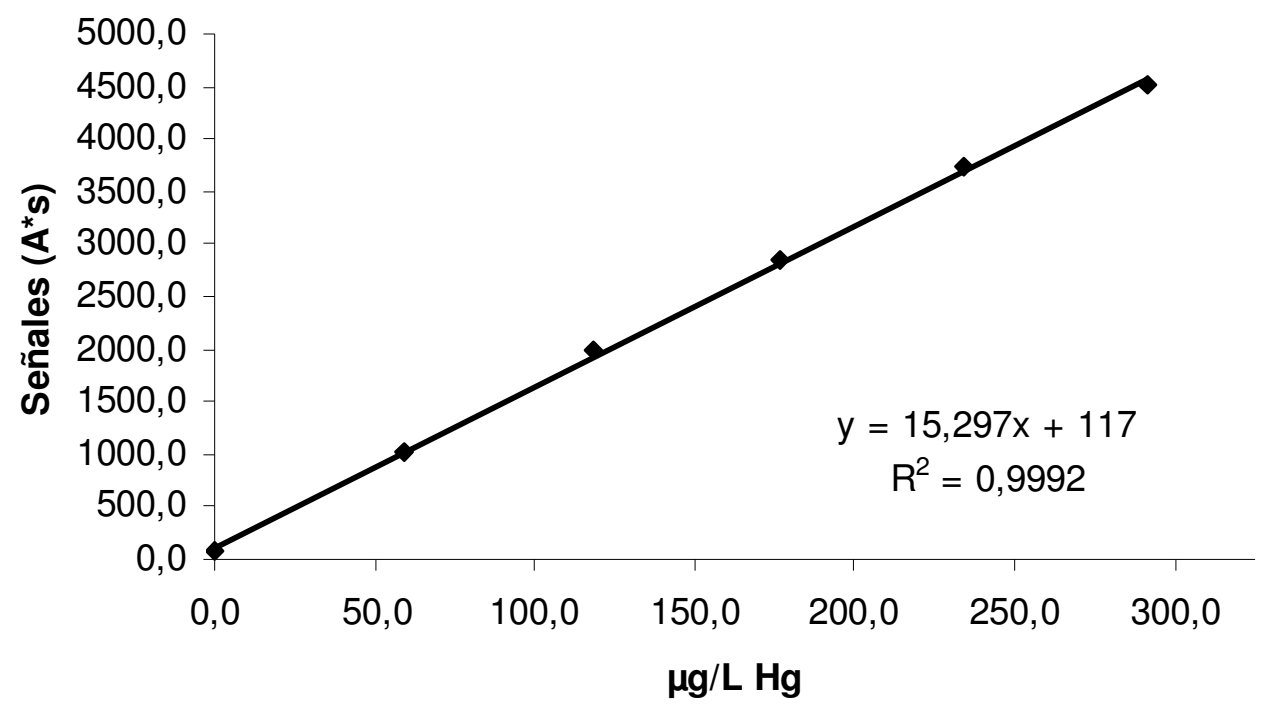

Figura 6. Linealidad del método de determinación de $\mathrm{Hg}^{2+}$.

\section{Determinación de los límites de detección}

Para establecer el límite de detección se utilizó el método de la recta de calibrado [12]. Al multiplicar este valor por $10 / 3$ se obtiene el valor del límite de cuantificación. El estudio fue hecho por quintuplicado y en la Tabla 1 se resumen los resultados obtenidos, mientras que en la Fig. 2 se observa una de las rectas de calibrado utilizadas para la determinación de estos límites.

Tabla 1. Limites de detección y cuantificación para la determinación de mercurio por análisis de redisolución potenciométrica PSA, $\mathrm{n}=5$.

\begin{tabular}{lc}
\hline \multicolumn{1}{c}{ Parámetro } & Magnitud \\
\hline Límite de detección & $5 \mu \mathrm{g} / \mathrm{L}$ \\
Límite de cuantificación & $17 \mu \mathrm{g} / \mathrm{L}$ \\
\hline
\end{tabular}

\section{Investigación por realizar}

En este punto de la investigación, se tiene completamente optimizado el método para la determinación de mercurio. Resta por estudiar el efecto de las posibles interferencias, así como los posibles métodos de mineralización de las muestras, 
tales que aseguren que no se pierda el mercurio debido a su alta volatilidad. Posteriormente, se procederá a validar la exactitud del método utilizando espectroscopia de absorción atómica, para finalmente aplicar la metodología al análisis de muestras medioambientales.

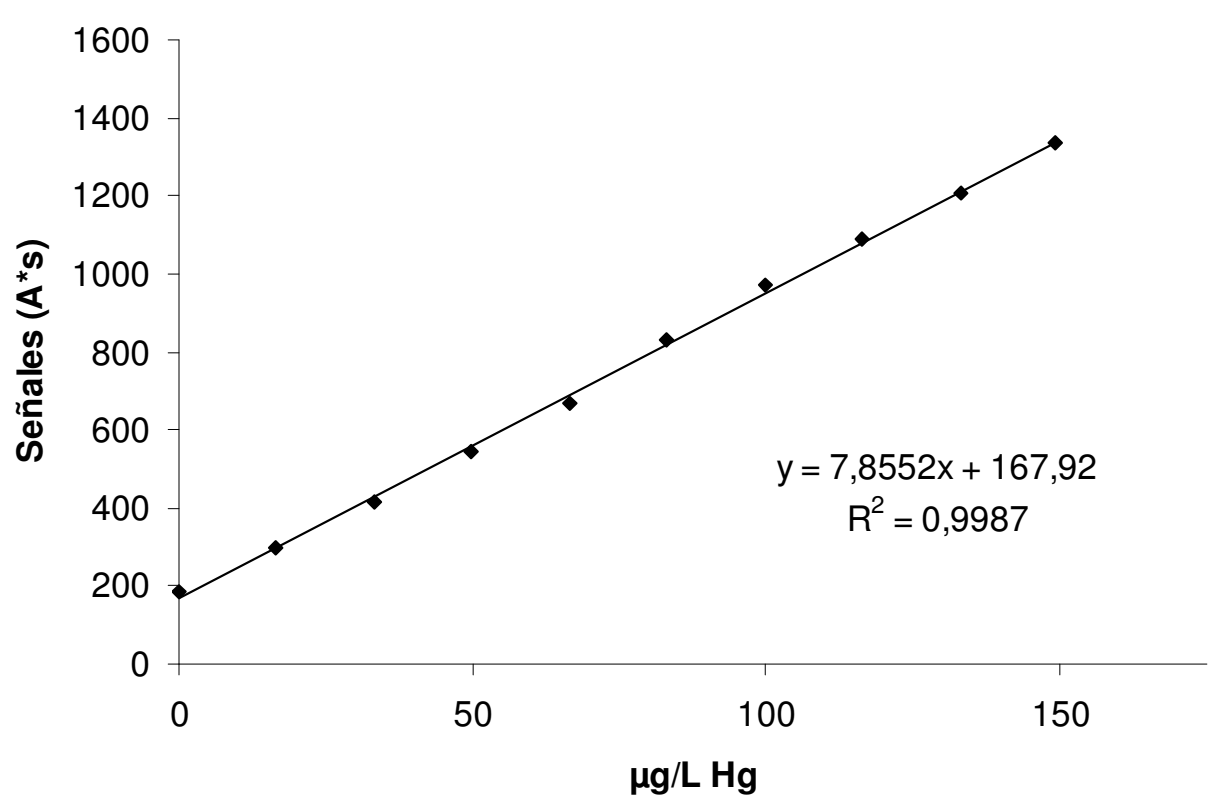

Figura 7. Recta de calibrado utilizada para la determinación del limite de detección por el método de Miller.

\section{Conclusiones}

Se establecieron las condiciones experimentales e instrumentales óptimas que permiten la determinación de trazas de mercurio, resumidas en la Tabla 2. El método presenta una sensibilidad y unos límites de detección y cuantificación (5 $\mu \mathrm{g} / \mathrm{L}$ y $17 \mu \mathrm{g} / \mathrm{L}$, respectivamente) apropiados para la determinación del metal en muestras ambientales. Actualmente se está trabajando en el método de mineralización a presión de las muestras a estudiar, así como en la eliminación de interferencias por medio de cromatografía de intercambio. 
Tabla 2. Condiciones experimentales seleccionadas para la determinación de mercurio en muestras ambientales.

\begin{tabular}{lc}
\hline Parámetro & Valor óptimo \\
\hline $\mathrm{Au}(\mathrm{III})$ & $50 \mathrm{mg} / \mathrm{L}$ \\
$\mathrm{KCl}$ & $0.0015 \mathrm{~mol} / \mathrm{L}$ \\
$\mathrm{H}_{2} \mathrm{SO}_{4}$ concentrado & $0.210 \mathrm{~mol} / \mathrm{L}$ \\
Potencial de electrólisis & $-250 \mathrm{mV}$ \\
Tiempo deposición de película & $420 \mathrm{~s}$ \\
Ámbito lineal & $0-250 \mu \mathrm{g} / \mathrm{L}$ \\
Limite detección & $5 \mu \mathrm{g} / \mathrm{L}$ \\
Limite cuantificación & $17 \mu \mathrm{g} / \mathrm{L}$ \\
\hline
\end{tabular}

\section{Agradecimientos}

Se agradece la colaboración brindada por el Servicio Alemán de Intercambio Académico (DAAD), por la beca de estudio de M. Miranda, y al Centro de Electroquímica y Energía Química (CELEQ) de la Universidad de Costa Rica por su apoyo a esta investigación.

\section{Establecimiento de un Método para la Determinación de Mercurio en Muestras Ambientales por Medio de la Técnica de Análisis por Redisolución potenciométrica (PSA)}

\section{Resumen}

El objetivo de este trabajo es el establecimiento de las condiciones instrumentales de análisis óptimas para la determinación y cuantificación de mercurio en muestras ambientales por medio de la técnica de Análisis por Redisolución Potenciométrica. La determinación de mercurio se fundamenta en la disolución de este en una película de oro previamente depositada sobre un electrodo de carbón vitrificado seguida de su redisolución por medio de un agente oxidante adicionado a la disolución de trabajo. Se realizaron ensayos con el fin de determinar la concentración de oxidante adicionado a la muestra, potencial de electrólisis, electrolito soporte y su concentración, tiempo de deposición de la película de oro, concentración de cloruro de potasio como fuente de iones $\mathrm{Cl}^{-}$, así como los límites de detección y cuantificación. Esto con el fin de determinar de forma precisa, reproducible y confiable la presencia de mercurio para su posterior cuantificación, previa mineralización por microondas y su preconcentración por cromatografía en columna de intercambio catiónico, con el fin de eliminar interferencias por presencia de iones metálicos. La cuantificación se realizó por el método de adiciones estándar. El estudio mostró un límite de detección de $5 \mu \mathrm{g} / \mathrm{L}$ y un límite de cuantificación de $17 \mu \mathrm{g} / \mathrm{L}$.

Palabras clave: Mercurio, stripping, PSA, ambiente, optimización, película de oro, limites de detección. 


\section{Referencias}

1. W.L. Clevenger, B.W. Smith, Winefordner, Critical Rev. Anal. Chem. 27 (1997) 1-26.

2. S. Manahan, Environmental Chemistry, 7a. edición, Lewis, Boca Ratón, U.S.A., 2000.

3. J.M. Estela, C. Tomás, A. Cladera, V. Cerdá, Critical Rev. Anal. Chem. 25 (1995) 91-141.

4. D. Jagner, Anal. Chim. Acta 105 (1979) 33-41.

5. C.H. Labar, Electroch. Acta 38 (1993) 807 - 813.

6. A.G. Fogg, J. Wang, Pure Appl. Chem. 71 (1999) 891- 897.

7. P. Ostapczuk, Anal. Chim. Acta 273 (1993) 35-40.

8. J. Wang, B. Tian, Anal. Chim. Acta 274 (1993) 1-6.

9. P. Manglano, R. Farré, A. Frigola, M.J. Lagarda, Ars. Pharmaceutica 44 (2003) 271-279.

10. R.D. Riso, M. Waeles, P. Monbet, Ch. Chaumery, Anal. Chim. Acta 410 (2000) 97-105.

11. E.P. Gil, P. Ostapczuk, Anal. Chim. Acta 293 (1994) 55-65.

12. J.C. Miller, J.N. Miller, Estadística para Química Analítica, 4a Edición, Addison Wesley Iberoamericana, S.A., España, 2002. 